\title{
Finger reduction of nasal bone fracture under local anesthesia: outcomes and patient reported satisfaction
}

Young-Jae Lee, Kyeong-Tae Lee, Jai-Kyong Pyon

Department of Plastic Surgery, Samsung Medical Center, Sungkyunkwan University School of Medicine, Seoul, Korea
Background: Closed reduction of the fracture under general or local anesthesia with elevators or forceps is widely used to treat nasal bone fractures. However, operating under general anesthesia increases the risk of morbidity and raises the cost of management. Furthermore, using forceps or elevators may cause undercorrection, new fractures, mucosal damage, and nasal hemorrhage. We therefore performed manual reduction under local anesthesia, using the little finger, to minimize the demerits of treatment under general anesthesia with forceps or elevators and aimed to assess functional and aesthetic outcomes, and patient satisfaction.

Methods: Patients who visited the plastic and reconstructive surgery department between November 2016 and November 2017 with nasal bone fractures and treated by a single surgeon were prospectively followed up. Patients with simple unilateral or bilateral nasal bone fractures were treated with bedside finger reduction under local anesthesia and patients with comminuted nasal bone or septal fractures were scheduled for closed reduction under general anesthesia.

Results: Of 84 patients, 28 met the inclusion criterion and underwent bedside finger reduction under local anesthesia. Twenty-seven patients $(96.4 \%)$ were successfully contacted via telephone for survey. Twenty-three $(85.2 \%)$ showed good and three (11.1\%) showed fair results. All 27 patients $(100 \%)$ were satisfied with their postoperative function and $25(92.6 \%)$ were satisfied with their postoperative aesthetic result. Twenty-five patients (92.6\%) preferred the finger reduction method under local anesthesia over closed reduction under general anesthesia.

Conclusion: Finger reduction under local anesthesia in patients with mild unilateral or bilateral nasal bone fractures is an easy and efficient procedure with high patient satisfaction and favorable postoperative functional and aesthetic outcomes.

Keywords: Acquired nose deformity / Bone fracture / Finger / Fracture / Local anesthesia / Nasal bone / Nose / Patient satisfaction / Reduction / Rhinoplasty

\section{INTRODUCTION}

Nasal bone fracture is the most common type of facial bone fracture. Closed reduction, which can be conducted under gen-

Correspondence: Jai-Kyong Pyon

Department of Plastic Surgery, Samsung Medical Center, Sungkyunkwan

University School of Medicine, 81 Irwon-ro, Gangnam-gu, Seoul 06351, Korea

E-mail: pspriest.pyon@samsung.com

Received October 2, 2018 / Revised December 1, 2018 / Accepted December 2, 2018 eral or local anesthesia, has been the primary treatment modality for such fractures. Closed reduction under general anesthesia generally involves admission and hospitalization, performing pre-operation tests for general anesthesia, and additional cost. Comorbidities related to use of general anesthesia should also be considered. Asch and Walsham forceps, Boies elevators, and blade handles are routinely used in the reduction of nasal bone fractures. However, closed reduction using these instru- 
ments is usually conducted blindly and may lead to undercorrection, new fractures, mucosal damage, and nasal hemorrhage. Finger reduction of nasal bone fractures under general anesthesia is well documented [1,2]. However, only a few studies have investigated the effectiveness of manual reduction conducted under local anesthesia, and they do not contain detailed reports on patient-assessed pain and satisfaction. We therefore conducted a prospective cohort study to evaluate the postoperative functional and aesthetic outcomes and patient satisfaction following finger reduction under local anesthesia.

\section{METHODS}

\section{Study population}

We conducted a prospective cohort study of 84 patients, each with a chief complaint of a fractured nose, treated at the plastic and reconstructive surgery department from November 2016 to November 2017. Fracture diagnosis was based on clinical history, plain radiograph of the nasal bones, facial bone computed tomography (CT), and physical examination. The inclusion criterion for bedside finger reduction under local anesthesia was simple unilateral or bilateral nasal bone fracture with apparent asymmetry. The exclusion criterion was apparent symmetry with no prominent fracture or deviation on plain $\mathrm{X}$ ray and facial bone CT. Patients with comminuted nasal bone or septal fractures were scheduled for closed reduction under general anesthesia and were also excluded from the study.

\section{Operation and evaluation method}

The finger reduction method is similar to the conventional closed reduction methods except that the reduction is done with the physician's fingers without using a nasal elevator, blade handle, or forceps. The patient is seated with head support in an examination chair in the clinic office. After filling the nasal cavity under the fractured area with gauze soaked in 1\% lidocaine and 1:100,000 epinephrine and waiting for 15 minutes, the physician used the volar side of the fifth finger to examine the fracture area from inside the nasal cavity. Aseptic polygloves and ointment were used to ease the movement of the examining finger. Depending on the findings from the plain radiograph and facial bone CT, the volar side of the finger was placed right below the fractured nasal bone and lifted laterally or superiorly as if done by an instrument [3]. The end-point of reduction was when bony continuity was felt with the finger and symmetry was restored. If the fracture was adjudged to be overcorrected, it was readjusted by applying counterpressure on the raised fractured bone with the other fingers. After the reduction was completed and nasal bleeding was evaluated, inter- nal fixation was performed using Merocel (Medtronic Inc., Minneapolis, MI, USA) and Denver Splint (Summit Medical Inc., St. Paul, MI, USA) was used for external fixation and protection. Pain was assessed using the visual analog scale (VAS), with values from 0 to 10 ( 0 , no pain; 10 , unbearable pain). All procedures were performed by a single physician (YJL), a plastic and reconstructive surgery resident, under the supervision of the advisor.

\section{Patient follow-up}

The patients were followed up 5 days later in the clinic office, with nasal lateral and Water's view plain radiographs and facial bone CT taken just before the appointment. After evaluating the radiographs and CT scans, physical examination was conducted, and the symmetry and reduction state were evaluated.

If a result was poor or unchanged, with asymmetry and insufficient reduction or collapsed nasal vault, the result was marked as "poor" and the patient was scheduled for closed reduction under general anesthesia.

If a result was fair, with symmetry but with insufficient reduction, insufficient nasal vault shape, and unsatisfactory alignment seen on radiograph or CT, the result was marked as "mild" and the patient was sent home after Denver splint reapplication.

If a result was good, with appropriate correction of the overall nasal vault, normal to suboptimal alignment, and apparent symmetry, the result was marked as "good" and the patient was sent home after Denver splint reapplication.

The patients were followed up for at least 6 months after nasal reduction and contacted by telephone for survey using a structured questionnaire. Patients were asked to compare the symmetry of their nose before and after the treatment using a scale of 1 (very asymmetric) to 4 (very symmetric), and to assess their overall functional (breathing) and aesthetic satisfaction using a scale of 1 (very poor) to 10 (very satisfied). The patients were also asked if they would prefer the finger reduction method under local anesthesia to closed reduction under general anesthesia if they had a similar trauma and needed treatment.

\section{Statistical analysis}

The data set was organized in Excel (Microsoft Corp., Redmond, WA, USA). All analyses were performed using SPSS ver. 13.0 (SPSS Inc., Chicago, IL, USA). The results are presented as mean \pm standard error of the mean.

\section{RESULTS}

Of the 84 patients who visited the clinic with nasal bone frac- 
tures, 28 met the inclusion criterion and underwent bedside finger reduction under local anesthesia. Twenty-seven patients (96.4\%) were successfully contacted by telephone for the study. Follow-up was carried out for a minimum of 6 months following nasal reduction.

Regarding patient demographics, 17 patients (63\%) were male and $10(37 \%)$ were female. Patients were aged 11-66 years (average, 32.33 years). Patients presented to our unit on an average of 4.7 days (range, 1 to 18 days) following injury. Fourteen patients (51.9\%) had L1 fractures, 12 (44.4\%) had L2 fractures, and 1 (3.7\%) had F2 fracture. The average follow-up period for the survey was 13.2 months (range, 9 to 19 months) after treatment (Table 1).

At treatment, the average VAS score was $6.19 \pm 0.38$, and three patients (11.1\%) had minimal bleeding (Fig. 1). After treatment and postoperative follow-up, 23 patients (85.2\%) showed good results (Figs. 2-5) and three patients (11.1\%) showed mild results (Fig. 6). One patient (3.7\%) had a poor result and was rescheduled for closed reduction under general anesthesia. After follow-up with the questionnaires, the average scores assigned for symmetry by the patients were $2.04 \pm 0.13$ before treatment and $3.76 \pm 0.08$ after treatment (Fig. 7). The average satisfaction scores for functional and aesthetic outcomes were $4.89 \pm 0.06$ and $4.67 \pm 0.12$, respectively (Fig. 8). All 27 patients (100\%) were

Table 1. Demographics

\begin{tabular}{lc}
\hline Variable & Value \\
\hline Sex & $17(63)$ \\
Male & $10(37)$ \\
Female & \\
Fracture grade & $14(51.9)$ \\
L1 & $12(44.4)$ \\
L2 & 0 \\
F1 & $1(3.7)$ \\
F2 & \\
Unilaterality & $7(25.9)$ \\
Right & $7(25.9)$ \\
Left & $13(48.1)$ \\
Bilateral & \\
Nasal bleeding & $3(11.1)$ \\
Bleeding & $24(88.9)$ \\
No bleeding & $32.33(11-66)$ \\
Age (yr) & $4.74(1-18)$ \\
Treatment time after trauma (day) & $13.2(9-19)$ \\
\hline Follow-up time (mo) &
\end{tabular}

Values are presented as number (\%) or average (range).

L1, lateral impact, unilateral nasal vault fracture; L2, lateral impact, bilateral nasal vault fracture; F1, frontal impact, nasal tip fracture; F2, frontal impact, nasal tip, and nasal septum fracture. satisfied with their functional outcome and 25 patients (92.6\%) were satisfied with their aesthetic result. When asked what method of treatment they would prefer if they were treated for the same kind of trauma again, 25 patients $(92.6 \%)$ said they would prefer the finger reduction method under local anesthesia and only two patients (7.4\%) said they would prefer closed reduction under general anesthesia because they were afraid of the pain (Table 2).

\section{DISCUSSION}

Patients with nasal bone fractures usually visit the plastic surgery clinic. Closed reduction of nasal bone fractures is a widely accepted treatment modality aimed at using a minimally invasive method to achieve good long-term results. Although reduction methods involving the use of forceps, elevators, and handles are widely reported in medical literature [4-9], some studies describe methods where fractured bones are repositioned using instruments and fine-adjusted externally by hand [10]. Although the use of fingers for the manipulation and reduction of nasal bone fractures is not common, satisfactory outcomes have been reported with this technique under general anesthesia $[2,3,11]$. However, evaluation of patient tolerance and satisfaction of this technique under local anesthesia is not well documented.

The use of fingers for manipulation and reduction is advantageous as it minimizes damage to the nasal mucosa and reduces the incidence of nasal hemorrhages which could occur with the use of an instrument for reduction [1]. Nasal mucosal injury with bleeding is commonly seen after closed reduction with an instrument and can be a factor in the development of mucosal contracture and deformity over time. Many different approaches have being proposed to reduce mucosal injury during reduction but they are more invasive than conventional methods

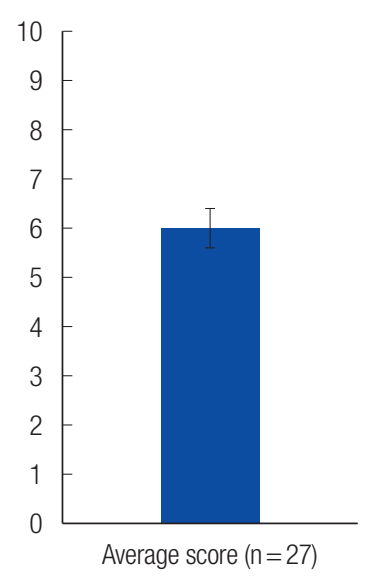

Fig. 1. Average visual analog scale score at treatment. 

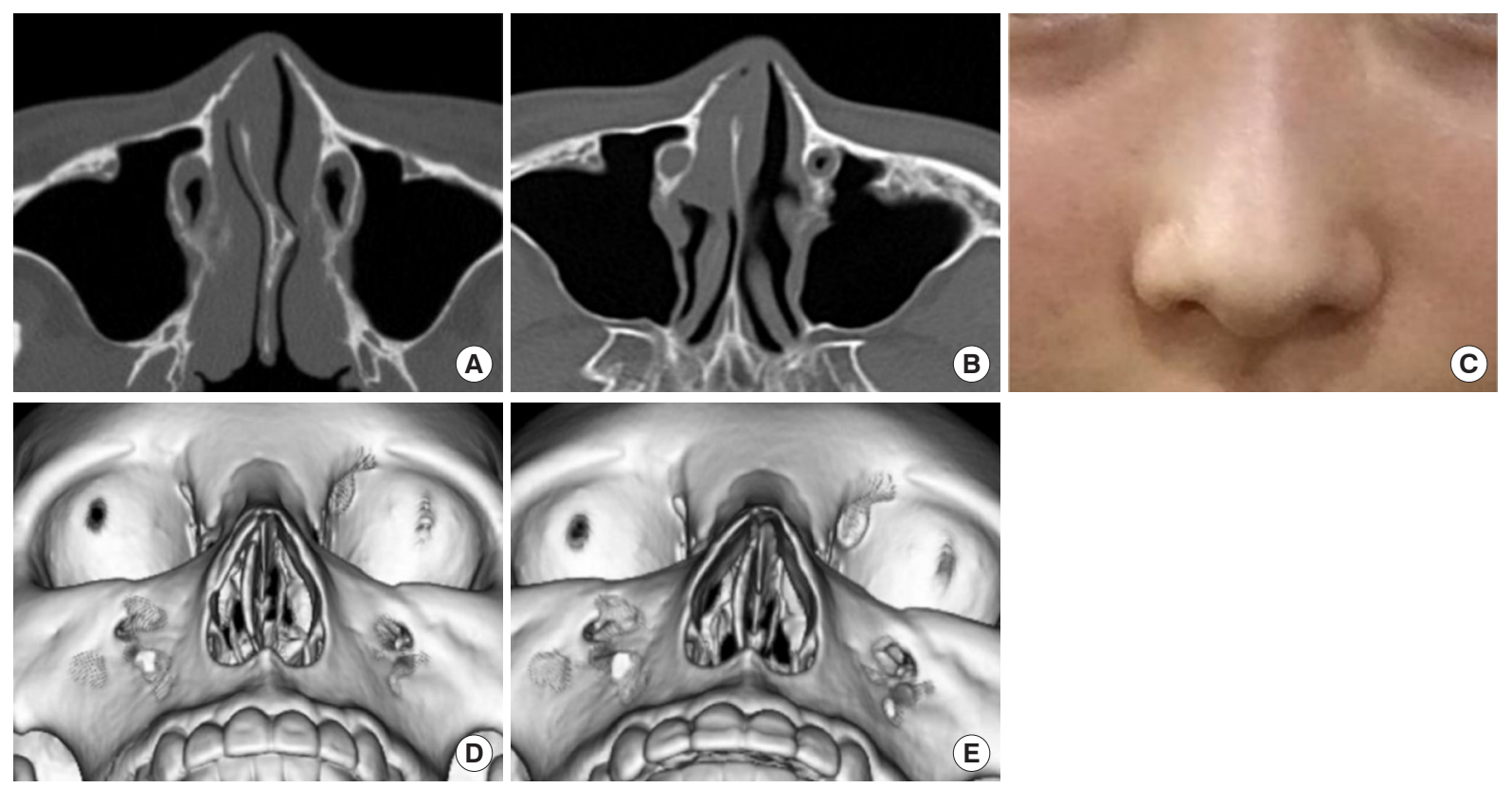

Fig. 2. (A) A 27-year-old female visited the clinic with a right unilateral nasal bone fracture (L1 grade: lateral impact, unilateral nasal vault fracture) 1 day after trauma. She was treated with manual finger reduction on the clinic chair; merocel was applied with Denver splint. (B) Five days after treatment, merocele and Denver splint was removed, and satisfactory reduction was seen on the facial bone computed tomography. (C) Symmetry is seen on the photograph taken 10 months after treatment. (D) Three-dimensional reconstruction model of fracture before treatment. (E) Three-dimensional reconstruction model of fracture 5 days after treatment.
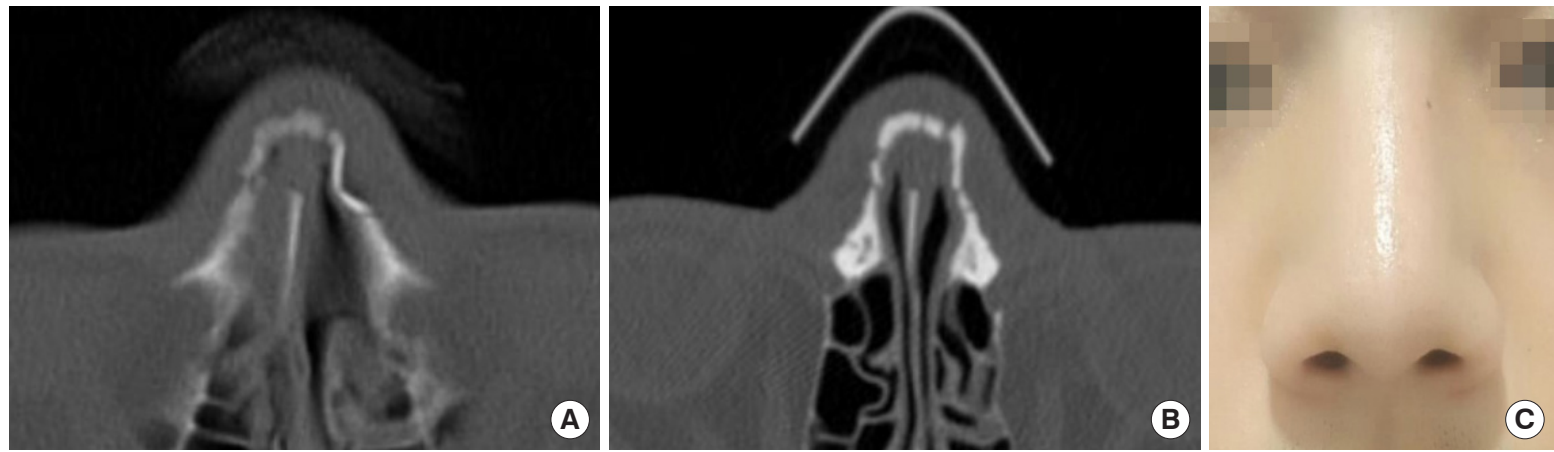

Fig. 3. (A) A 25-year-old male patient visited the clinic with a bilateral nasal bone fracture (L2 grade: lateral impact, bilateral nasal vault fracture) 6 days after trauma. The fracture was treated using manual finger reduction. (B) Satisfactory reduction was seen on computed tomography 8 days after the treatment. (C) Symmetry is seen on the photograph taken 16 months after the treatment.
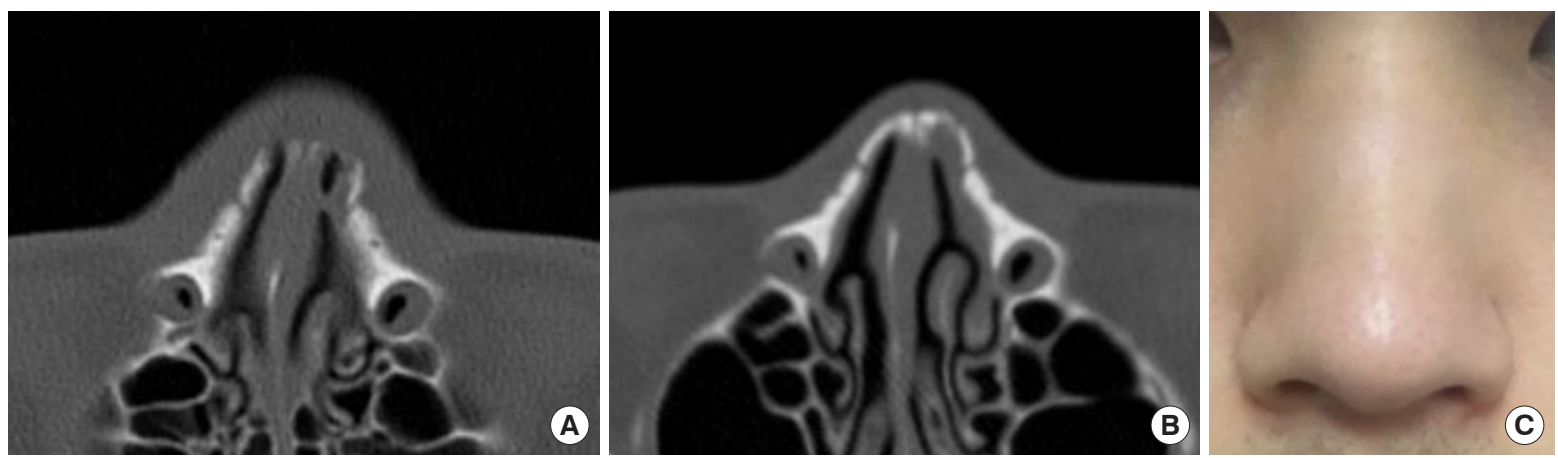

Fig. 4. (A) A 23-year-old male patient visited the clinic with a bilateral nasal bone fracture (L2 grade: lateral impact, bilateral nasal vault fracture) 3 days after trauma. The fracture was treated using manual finger reduction. (B) Satisfactory reduction was seen on computed tomography 8 days after the treatment. (C) Symmetry is seen on the photograph taken 10 months after the treatment. 

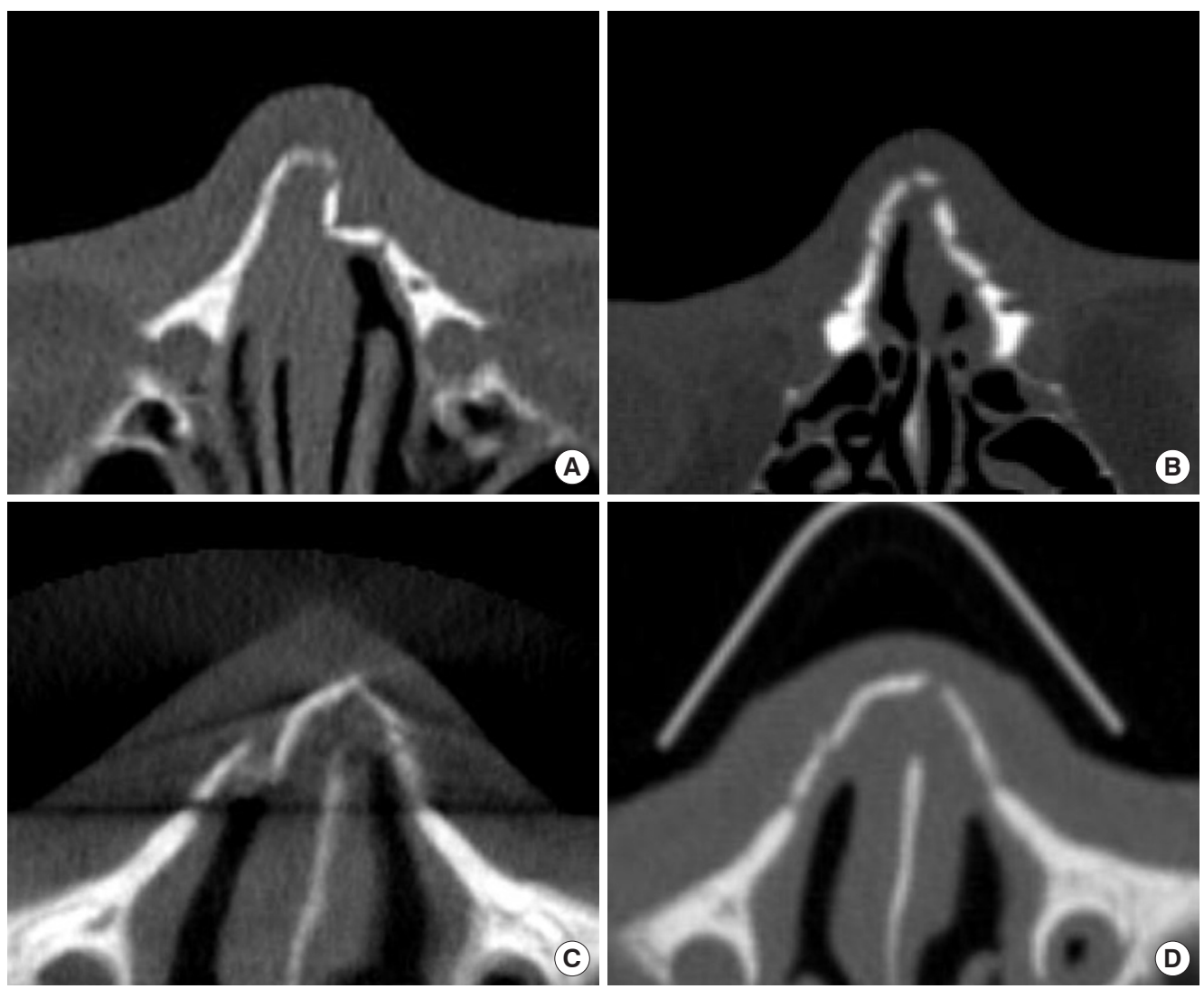

Fig. 5. (A) A 22-year-old male patient visited the clinic with a unilateral nasal bone fracture (L1 grade: lateral impact, unilateral nasal vault

fracture) 2 days after trauma. (B) Computed tomography 5 days after the treatment. (C) A 29-year-old male patient visited the clinic with a bilateral nasal bone fracture (L2 grade: lateral impact, bilateral nasal vault fracture) 2 days after trauma. (D) Computed tomography 3 days after the treatment.

Postoperative result

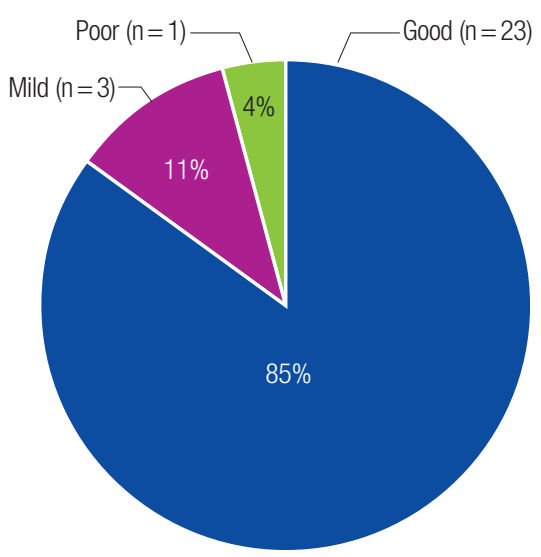

Fig. 6. Postoperative result on follow-up.

Table 2. Treatment preference of patients

\begin{tabular}{lc}
\hline Treatment choice & No. (\%) \\
\hline Manual finger reduction under local anesthesia & $25(92.6)$ \\
Reduction under general anesthesia & $2(7.4)$ \\
\hline
\end{tabular}

Patients preferred manual finger reduction under local anesthesia to reduction under general anesthesia, when asked what method of treatment they would prefer if they were to undergo the same trauma again.

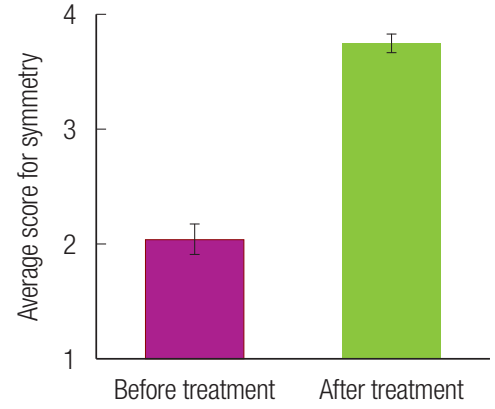

Fig. 7. Patient-assessed symmetry before and after treatment $(n=27)$.

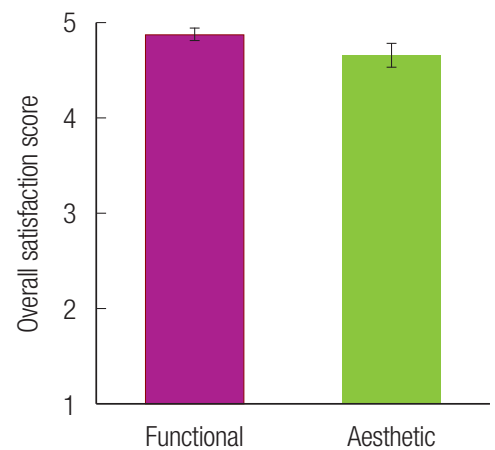

Fig. 8. Patient satisfaction for functional and aesthetic results $(n=27)$. 
[12]. In this study, following finger reduction, bleeding occurred in only three patients (11.1\%) and the bleeding was minimal in all three cases.

Studies show that prompt reduction increases the likelihood of favorable results [13-16], and it should be performed 3-10 days after trauma when the swelling has subsided [1]. In this study, finger reduction was performed on an average of 4.7 days following injury. All patients were able to receive treatment on the day of their clinic visit because finger reduction under local anesthesia can be performed in the clinic examination chair with no need for blood tests or an operation room, unlike in procedures performed under general anesthesia.

This technique allows for easy determination of the exact condition of the fractured bone and of the exact area to be manually reduced because the nasal mucosa is thin and directly attached to the bone [3]. Furthermore, the strength and direction of the force applied to the fractured area can be easily regulated by the physician with direct palpation. Finger reduction also causes minimal swelling and allows for easy evaluation of the result of the procedure. The limitations to this method include the different finger sizes of physicians and the different nasal cavity sizes of patients. In this study, the physician's little fingers were small enough for insertion into the nasal cavities, for palpation, and treatment of all the cases. However, this method would be difficult to execute if the physician had relatively bigger little fingers, or if the patients had relatively small nasal cavities. Further, patient selection was limited to fractures not involving the nasal septum, as reduction of severe nasoethmoid fractures or nasal septum fractures would be difficult to treat manually.

Although the average VAS score at treatment was 6.19 (range, 0 to 10$), 25$ of the 27 patients said that the pain was brief and tolerable and that they would prefer finger reduction treatment under local anesthesia to closed reduction under general anesthesia if they have a similar trauma in the future. This may be because the pain felt by patients is only a sharp but tolerably short pain, and because of uncertainty of a better result with treatment under general anesthesia.

This study does not seek to discourage the use of reduction methods with instruments or reduction under general anesthesia, as they have many advantages. However, we reckon that preliminary examination and initial attempt at fracture reduction using the finger can facilitate better assessment of the fracture and lead to a more accurate and tolerable reduction. In addition, as patients are generally anxious about treatment with instruments under local anesthesia and about undergoing surgery under general anesthesia, this method can be an ideal initial treatment method.
Finger reduction of nasal bone fractures under local anesthesia saves time and space, has no need for instruments, and offers high patient tolerance and satisfaction. Therefore, it can be a useful initial treatment for nonsevere nasal bone fractures encountered in daily clinical practice.

\section{NOTES}

\section{Conflict of interest}

No potential conflict of interest relevant to this article was reported.

\section{Ethical approval}

The study was performed in accordance with the principles of the Declaration of Helsinki. Written informed consent was obtained.

\section{Patient consent}

The patients provided written informed consent for the publication and the use of their images.

\section{ORCID}

Young-Jae Lee https://orcid.org/0000-0002-4130-0697

Kyeong-Tae Lee https://orcid.org/0000-0002-9070-9296

Jai-Kyong Pyon https://orcid.org/0000-0002-6302-3002

\section{REFERENCES}

1. Rubinstein B, Strong EB. Management of nasal fractures. Arch Fam Med 2000;9:738-42.

2. Love RL. Nasal fractures: patient satisfaction following closed reduction. N Z Med J 2010;123:45-8.

3. Ichida M, Komuro Y, Koizumi T, Shimizu A, Yanai A. A repositioning technique for nasal fracture using the little finger. J Craniofac Surg 2008;19:1512-7.

4. Bowerman E. Fractures of the middle third of the facial skeleton. In: Rowe NL, Williams JL, editors. Maxillofacial injuries. New York: Churchill Livingstone; 1985. p. 363-433.

5. Aloin HA. A propose du traitement des fractures du nez. note de tequnique chirurgicale. J Fr Otorhinolaryngol 1952;1:302.

6. Humber PR, Horton CE. Trauma to the nose. In: Stark RB, editor. Plastic surgery of the head and neck. New York: Churchill Livingstone; 1987. p. 390-401.

7. Tremolet de Villers Y. Nasal fractures. J Trauma 1975;15:31927.

8. Tajima S. Treatment of facial bone fractures. 2nd ed. Tokyo: Kokuseido Publishing; 1999. p. 143-74.

9. Paul N. Manson: facial fractures. In: Sherrell JA, Robert W, 
Beasley C, editors. Plastic Surgery. 5th ed. Philadelphia: Lippincott-Raven; 1997. p. 383-411.

10. Kurihara K, Kim K, Miyata K. Clinical study of new nasal bone fractures. Jpn J Plast Surg 1990;33:113-20.

11. Rajapakse Y, Courtney M, Bialostocki A, Duncan G, Morrissey G. Nasal fractures: a study comparing local and general anaesthesia techniques. ANZ J Surg 2003;73:396-9.

12. Kim HS, Suh HW, Ha KY, Kim BY, Kim TY. The usefulness of the endonasal incisional approach for the treatment of nasal bone fracture. Arch Plast Surg 2012;39:209-15.
13. Goode RL, Spooner TR. Management of nasal fractures in children: a review of current practices. Clin Pediatr (Phila) 1972;11:526-9.

14. Fomon S, Schattner A, Bell JW, Kleinfeld L, Lewy R. Management of recent nasal fractures. AMA Arch Otolaryngol 1952; 55:321-42.

15. Gillies H, Kilner T. Modern technique in treatment. Lancet 1929;1:147-9.

16. Safian J, Tamerin J. Recent fractures of the nose. Am J Surg 1936;31:10-23. 